\title{
ReasonBERT: Pre-trained to Reason with Distant Supervision
}

\author{
Xiang Deng*1, Yu Su${ }^{1}$, Alyssa Lees ${ }^{2}$, You $\mathrm{Wu}^{2}$, Cong $\mathrm{Yu}^{2}$, and Huan Sun*1 \\ ${ }^{1}$ The Ohio State University, Columbus, $\mathrm{OH}$ \\ \{deng.595, su.809, sun.397\} dosu. edu \\ ${ }^{2}$ Google Research, New York, NY \\ \{alyssalees, wuyou, congyu\} dgoogle.com
}

\begin{abstract}
We present ReasonBERT, a pre-training method that augments language models with the ability to reason over long-range relations and multiple, possibly hybrid, contexts. Unlike existing pre-training methods that only harvest learning signals from local contexts of naturally occurring texts, we propose a generalized notion of distant supervision to automatically connect multiple pieces of text and tables to create pre-training examples that require long-range reasoning. Different types of reasoning are simulated, including intersecting multiple pieces of evidence, bridging from one piece of evidence to another, and detecting unanswerable cases. We conduct a comprehensive evaluation on a variety of extractive question answering datasets ranging from single-hop to multi-hop and from text-only to table-only to hybrid that require various reasoning capabilities and show that ReasonBERT achieves remarkable improvement over an array of strong baselines. Fewshot experiments further demonstrate that our pre-training method substantially improves sample efficiency. ${ }^{1}$
\end{abstract}

\section{Introduction}

Recent advances in pre-trained language models (LMs) have remarkably transformed the landscape of natural language processing. Pre-trained with self-supervised objectives such as autoregressive language modeling (Radford and Narasimhan, 2018; Radford et al., 2019; Brown et al., 2020) and masked language modeling (MLM) (Devlin et al., 2019; Liu et al., 2019b; Joshi et al., 2020), LMs encode a great deal of knowledge about language and significantly boost model performance on a wide range of downstream tasks (Liu et al., 2019a; Wang et al., 2019a,b) ranging from spell checking

\footnotetext{
*Corresponding authors.

${ }^{1}$ Our code and pre-trained models are available at https : //github.com/sunlab-osu/ReasonBERT.
}

(Awasthi et al., 2019) to sentiment analysis (Xu et al., 2019) and semantic parsing (Rongali et al., 2020), just to name a few.

Existing self-supervised objectives for LM pretraining primarily focus on consecutive, naturally occurring text. For example, MLM enables LMs to correctly predict the missing word "daughters" in the sentence "Obama has two _, Malia and Sasha." based on the local context and the knowledge stored in the parameters. However, many tasks require reasoning beyond local contexts: multi-hop question answering (QA) (Yang et al., 2018; Welbl et al., 2018) and fact verification (Jiang et al., 2020) require reasoning over multiple pieces of evidence, hybrid QA (Chen et al., 2020) requires simultaneously reasoning over unstructured text and structured tables, and dialogue systems require reasoning over the whole dialogue history to accurately understand the current user utterance (Andreas et al., 2020).

To address this limitation in existing LM pretraining, we propose ReasonBERT, a pre-training method to augment LMs for explicitly reasoning over long-range relations and multiple contexts. ReasonBERT pairs a query sentence with multiple relevant pieces of evidence drawn from possibly different places and defines a new LM pre-training objective, span reasoning, to recover entity spans that are masked out from the query sentence by jointly reasoning over the query sentence and the relevant evidence (Figure 1). In addition to text, we also include tables as evidence to further empower LMs to reason over hybrid contexts.

One major challenge in developing ReasonBERT lies in how to create a large set of query-evidence pairs for pre-training. Unlike existing self-supervised pre-training methods, examples with complex reasoning cannot be easily harvested from naturally occurring texts. Instead, we draw inspiration from distant supervision (Mintz et al., 2009a), which assumes 
The [QUESTION], was the third edition of the [QUESTION], the premier beach soccer competition contested between European men's national teams, known as the [QUESTION] at the time, occurring annually since its establishment in 1998.

The Euro Beach Soccer League (EBSL) is the premier competition in beach soccer contested between European

men's national teams

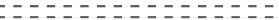

Metal Blade Records Unanswerable

Awaken the Guardian is the third studio album by [QUESTION] band [QUESTION], released in 1986 through [QUESTION].

Fates Warning is an American progressive metal band,

formed in 1982 by vocalist John Arch, guitarists Jî̀

Matheos and Victor Arduini, bassist Joe DiBiase, and

drummer Steve Zimmerman in Hartford, Connecticut.
Originally called the European Pro Beach Soccer League until 2004, the competition has been held annually since its establishment in 1998
Hybrid, with bridging entity

\begin{tabular}{|c|c|c|c|}
\hline Title & Author & Work & Year \\
\hline "Morgana" & Lord of the Lost & Thornstar & 2018 \\
\hline "Morgan Le Fay" & Lovespoon & Lovespoon & 2011 \\
\hline "Fata Morgana" & Fates Warning & $\frac{\text { Awaken the }}{\text { Guardian }}$ & 1986 \\
\hline
\end{tabular}

Figure 1: Examples of our pre-training data acquired via distant supervision, which covers a wide range of topics with both textual and tabular evidence. For each query sentence (in black), we first select two pairs of entities (underlined) to find two pieces of evidence (in grey) via distant supervision. We then randomly mask one entity from each selected pair and aim to recover it by reasoning over the evidence. Note that the two selected pairs may share a common entity; in case this entity is masked, we can mimic different types of multi-hop reasoning, e.g., intersection (Ex. 1) and bridging (Ex. 2). To simulate unanswerable cases, we additionally mask one entity (in blue) that does not exist in the evidence. Figure best viewed in color.

that "any sentence containing a pair of entities that are known to participate in a relation is likely to express that relation," and generalize it to our setting of multiple pieces of evidence from text and tables. Specifically, given a query sentence containing an entity pair, if we mask one of the entities, another sentence or table that contains the same pair of entities can likely be used as evidence to recover the masked entity. Moreover, to encourage deeper reasoning, we collect multiple pieces of evidence that are jointly used to recover the masked entities in the query sentence, allowing us to scatter the masked entities among different pieces of evidence to mimic different types of reasoning. Figure 1 illustrates several examples using such distant supervision. In Ex. 1, a model needs to check multiple constraints (i.e., intersection reasoning type) and find "the beach soccer competition that is established in 1998." In Ex. 2, a model needs to find "the type of the band that released Awaken the Guardian," by first inferring the name of the band "Fates Warning" (i.e., bridging reasoning type).

We first replace the masked entities in a query sentence with the [QUESTION] tokens. The new pre-training objective, span reasoning, then extracts the masked entities from the provided evidence. We augment existing LMs like BERT (Devlin et al., 2019) and RoBERTa (Liu et al., 2019b) by continuing to train them with the new objective, which leads to ReasonBERT, a new LM with better reasoning capabilities. Then query sentence and textual evidence are encoded via the LM. When tabular evidence is present, we use the structure-aware transformer TAPAS (Herzig et al., 2020) as the encoder to capture the table structure.

We evaluate ReasonBERT on the extractive QA task, which is arguably the most representative task requiring reasoning about world knowledge. We conduct a comprehensive evaluation using a variety of popular datasets: MRQA (Fisch et al., 2019), a single-hop QA benchmark including six datasets from different domains; HotpotQA (Yang et al., 2018), a multi-hop QA dataset; NQTables, a subset of the Natural Questions dataset (Kwiatkowski et al., 2019) where answers can be found in tables; and HybridQA (Chen et al., 2020), a hybrid multi-hop QA dataset that requires reasoning over both tables and text. Under the few-shot setting, ReasonBERT substantially outperforms the baselines in almost all datasets, demonstrating that the reasoning ability learned from pre-training can easily transfer to downstream QA tasks and generalize well across domains. Under the full-data setting, ReasonBERT obtains substantial gains in multihop and hybrid QA datasets. Despite its simple model architecture, ReasonBERT achieves similar or better performance compared with more sophisticated state-of-the-art models for each dataset. 


\section{Background}

Language model pre-training. Existing pretraining objectives such as MLMs (Devlin et al., 2019; Joshi et al., 2020) tend to implicitly memorize the learned knowledge in the parameters of the underlying neural network. In this work, we aim to augment pre-training by encouraging a model to reason about (instead of memorizing) world knowledge over the given contexts.

Extractive question answering. To measure a model's reasoning ability about world knowledge, we select extractive QA as a downstream task, which is perhaps one of the most representative tasks for this purpose. Given a question $q$ and provided evidence $E$, an extractive QA model $p_{\theta}(a \mid q, E)$ aims to select a contiguous span $a$ from $E$ that answers the question, or output a special token if $E$ is not sufficient to answer the question.

Our approach, ReasonBERT, is inspired by this formulation and extends it to language model pretraining. The challenge in defining such a selfsupervised task is in the creation of questionevidence pairs from unlabeled data. Moreover, we aim for a generic approach that works for a wide range of extractive QA settings including singlehop and multi-hop reasoning, hybrid contexts with both unstructured texts and structured tables, as well as few-shot settings. We discuss how to address the challenge and achieve this goal in the next two sections.

\section{Distant Supervision (DS) for Pre-training}

We use English Wikipedia as our data source for pre-training. We first extract sentences and tables from Wikipedia pages and then identify salient spans (such as named entities) from them. We apply the idea of distant supervision and match the sentences and tables to form query-evidence pairs, which are used to create pre-training examples.

\subsection{Data Collection}

Text. We first extract paragraphs from Wikipedia pages and split them into sentences. We consider named entities including both real-world entities (e.g., person, location) and temporal and numeric expressions (e.g., date and quantity) as potential answer entities for pre-training. We first identify real-world entities using existing hyperlinks. Since Wikipedia pages generally do not contain links to themselves, we additionally detect such selfmentions by searching the names and aliases of the topic entity for each page. Temporal and numeric expressions are identified using an existing NER tool $^{2}$.

Tables. We extract tables that are labeled as <wikitable $>$ from Wikipedia, and only consider tables with no more than 500 cells. First, real-world entities are detected using existing hyperlinks. Unlike our method employed for textual sentences, we do not use traditional NER tools here as they are not tailored to work well on tables. Instead, for a cell that does not contain hyperlinks, we match the complete cell value with sentences that are closely related to the table, sourced either from the same page or a page containing a hyperlink pointing to the current page. If the matched span in the sentence contains a named entity, we consider the same entity as being linked to the cell as well. Otherwise we consider this cell as a unique entity in the table.

Please see Appendix A.1 for details about the tools and resources we use.

\subsection{Query-Evidence Pairing via DS}

As described in Section 2, a standard QA sample is composed of a question, an answer and evidence. The model infers the relationship between the answer and other entities in the question, and extracts it from the evidence. In this work, we try to simulate such samples in pre-training. Given a sentence with entities, it can be viewed as a question by masking some entities as answers for prediction. The key issue is then how to find evidence that contains not only the answer entity, but also the relational information for inference. Here we borrow the idea of distant supervision (Mintz et al., 2009b).

Given a sentence as a query, we first extract pairs of entities in it. For each entity pair, we then find other sentences and tables that also contain the same pair as evidence. Since we do not have the known relation constraint in the original assumption of distant supervision, we use the following heuristics to collect evidence that has high quality relational knowledge about the entities and is relevant to the query. First, we only consider entity pairs that contain at least one real-world entity. For textual evidence, the entity pair needs to contain the topic entity of the Wikipedia page, which is more likely to have relations to other entities. For

\footnotetext{
${ }^{2}$ https://nlp. johnsnowlabs.com/
} 


\begin{tabular}{lcccc}
\hline Setting & \# queries & \# sent. & \# tab. & \# ent. pairs \\
\hline Text-only & $7.6 \mathrm{M}$ & $8.4 \mathrm{M}$ & - & $5.5 \mathrm{M}$ \\
Hybrid & $3.2 \mathrm{M}$ & $4.3 \mathrm{M}$ & $0.9 \mathrm{M}$ & $6.0 \mathrm{M}$ \\
\hline
\end{tabular}

Table 1: Statistics about the pre-training data.

\begin{tabular}{lccc}
\hline Setting & All the same & One different & All different \\
\hline Text-only & $50 \%$ & $30 \%$ & $20 \%$ \\
Hybrid & $60 \%$ & $8 \%$ & $32 \%$ \\
\hline
\end{tabular}

Table 2: Analysis of pre-training data quality with 50 examples for each setting. One different is when the relation between the selected entities is different from the relation expressed in the query sentence for of the two pieces of evidence.

tabular evidence, we consider only entity pairs that are in the same row of the table, but they do not need to contain the topic entity, as in many cases the topic entity is not present in the tables. In both cases, the query and evidence should come from the same page, or the query contains a hyperlink pointing to the evidence page. For tabular evidence, we also allow for the case where the table contains a hyperlink pointing to the query page.

\subsection{Pre-training Data Generation}

Given the query-evidence pairs, a naive way to construct pre-training examples is to sample a single piece of evidence for the query, and mask a shared entity as "answer", as in Glass et al. (2020). However, this only simulates simple single-hop questions. In this work, we construct complex pre-training examples that require the model to conduct multi-hop reasoning. Here we draw inspiration from how people constructed multi-hop QA datasets. Take HotpotQA (Yang et al., 2018) as an example. It first collected candidate evidence pairs that contain two paragraphs $(A, B)$, with a hyperlink from $A$ to $B$ so that the topic entity of $B$ is a bridging entity that connects $A$ and $B$. Crowd workers then wrote questions based on each evidence pair. Inspired by this process, we combine multiple pieces of evidence in each pre-training example and predict multiple masked entities simultaneously. The detailed process is described below. Figure 1 shows two examples. For more examples, please check Appendix A.1.

We start by sampling up to two entity pairs from the query sentence and one piece of evidence (sentence or table) for each entity pair. We then mask one entity in each pair as the "answer" to predict. The resulting pre-training examples fall into three categories: (1) Two disjoint entity pairs $\{(a, b),(c, d)\}$ are sampled from the query, and one entity from each pair, e.g., $\{a, c\}$, is masked. This is similar to a combination of two singlehop questions. (2) The two sampled entity pairs $\{(a, b),(b, c)\}$ share a common entity $b$, and $b$ is masked. The model needs to find two sets of entities that respectively satisfy the relationship with $a$ and $c$, and take an intersection (Type II in HotpotQA; see Ex. 1 in Figure 1). (3) The two sampled entity pairs $\{(a, b),(b, c)\}$ share a common entity $b$, and $\{b, c\}$ are masked. Here $b$ is the bridging entity that connects $a$ and $c$. The model needs to first identify $b$ and then recover $c$ based on its relationship with $b$ (Type I and Type III in HotpotQA; see Ex. 2 in Figure 1). We also mask an entity from the query that is not shown in the evidence to simulate unanswerable cases. All sampling is done randomly during pre-training.

\subsection{Data Statistics and Analysis}

We prepare pre-training data for two settings: (1) one with only textual evidence (text-only) and (2) the other including at least one piece of tabular evidence in each sample (hybrid). Some statistics of the collected data are summarized in Table 1. For the text-only setting, we extract approximately 7.6M query sentences, each containing 2 entity pairs that are matched with 3 different pieces of textual evidence on average. For the hybrid setting, we select approximately 3.2M query sentences, each containing 3.5 entity pairs, matched with 5.8 different pieces of evidence on average.

We also conduct an analysis of the pre-training data quality using 50 randomly sampled examples from each setting. We compare the query sentence and the evidence to see if they are expressing the same relation between the selected entities. Results are summarized in Table 2. We can see that in both settings, almost $70 \%$ of the examples have the desired characteristic that the evidence contains useful relational knowledge for recovering missing entities in the query sentence.

\section{Pre-training}

\subsection{Encoder}

For the text-only setting, we use the standard transformer encoder in BERT (Devlin et al., 2019). For settings where the input contains tables, we adopt the transformer variant recently introduced in TAPAS (Herzig et al., 2020), which uses extra token-type embeddings (indicating the row/column 
position of a token) to model the table structure.

\subsection{Span Reasoning Objective}

Now we describe our span reasoning objective, which can advance the reasoning capabilities of a pre-trained model.

Given a sample collected for pre-training as described in Section 3.3, we replace the masked entities $\mathcal{A}=\left\{a_{1}, \ldots, a_{n}\right\}(n \leq 3)$ in the query sentence $q$ with special [QUESTION] tokens. The task then becomes recovering these masked entities from the given evidence $E$ (concatenation of the sampled evidence). Specifically, we first concatenate $q, E$ and add special tokens to form the input sequence as [ [CLS ], $q,[\mathrm{SEP}], E]$, and get the contextualized representation $\mathbf{x}$ with the encoder. Since we have multiple entities in $q$ masked with [QUESTION], for each $a_{i}$, we use its associated [QUESTION] representation as a dynamic query vector $\mathbf{x}_{a_{i}}$ to extract its start and end position $s, e$ of $a_{i}$ in $E$ (i.e., question-aware answer extraction).

$$
\begin{aligned}
& P(s \mid q, E)=\frac{\exp \left(\mathbf{x}_{s}^{\top} \mathbf{S} \mathbf{x}_{a_{i}}\right)}{\sum_{k} \exp \left(\mathbf{x}_{k}^{\top} \mathbf{S} \mathbf{x}_{a_{i}}\right)} \\
& P(e \mid q, E)=\frac{\exp \left(\mathbf{x}_{e}^{\top} \mathbf{E} \mathbf{x}_{a_{i}}\right)}{\sum_{k} \exp \left(\mathbf{x}_{k}^{\top} \mathbf{E} \mathbf{x}_{a_{i}}\right)}
\end{aligned}
$$

Here $\mathbf{S}, \mathbf{E}$ are trainable parameters. $\mathbf{x}_{a_{i}}$ is the representation of special token [QUESTION] corresponding to $a_{i} ; \mathbf{x}_{k}$ is the representation of the $k$-th token in $E$. If no answer can be found in the provided evidence, we set $s, e$ to point to the [CLS ] token.

The span reasoning loss is then calculated as follows:

$$
L_{S R}=-\sum_{a_{i} \in \mathcal{A}}\left(\log P\left(s_{a_{i}} \mid q, E\right)+\log P\left(e_{a_{i}} \mid q, E\right)\right)
$$

We name this objective as span reasoning, as it differs from the span prediction/selection objectives in existing pre-training work such as SpanBert (Joshi et al., 2020), Splinter (Ram et al., 2021), and SSPT (Glass et al., 2020) in the following ways: (1) Unlike SpanBert and Splinter that use single contiguous paragraph as context, where the models may focus on local cues, we encourage the model to do long-range contextualization by including both query and evidence as input, which can come from different passages, and recovering the masked entities by grounding them on the evidence $E$. (2) Unlike SSPT, we improve the model's ability to reason across multiple pieces of evidence by including two disjoint pieces of evidence in a single sample and scattering the answer entities among

\begin{tabular}{lcccc}
\hline & MRQA & HotpotQA & NQTables & HybridQA \\
\hline \# train & 86136.5 & 88881 & 17112 & 62686 \\
\# dev & - & 1566 & 1901 & 3466 \\
\# test & 9704 & 7405 & 1118 & 3463 \\
\# evidence & 1 & 10 & 8.7 & 34.7 \\
\# tokens* & 374.9 & 89.1 & 289.6 & 156.3 \\
has text/table & $\mathbb{S} / \boldsymbol{X}$ & $\mathbb{S} \boldsymbol{X}$ & $\boldsymbol{X} / \boldsymbol{S}$ & $\mathbb{S} \boldsymbol{S}$ \\
\hline
\end{tabular}

Table 3: Dataset statistics. The statistics for MRQA are averaged over all 6 datasets. \# tokens* is the average number of tokens per evidence.

them to mimic different types of reasoning chains. (3) We mimic the scenario where a span cannot be inferred based on the given contexts, by masking entities in $q$ that do not appear in $E$, in which case the model is trained to select the special [CLS ] token.

\subsection{Final Objective}

We also include the masked language modeling (MLM) objective in pre-training to leverage other tokens in the input that are not entities. In particular, we randomly mask tokens that are not an entity or token in the header row for tables, and use an MLM objective to recover them. Following the default parameters from BERT, we use a masking probability of $15 \%$.

The final loss is the sum of span reasoning loss and masked language modeling loss. Following previous work (Glass et al., 2020; Herzig et al., 2020), we initialize with a pre-trained encoder, and extend the pre-training with our objectives. For the text part, we pre-train two models with BERTBase (denoted as ReasonBERTв) and RoBERTaBase (denoted as ReasonBERTR); for the table part, we use TAPAS-Base (denoted as ReasonBERTT). More implementation details of pre-training are included in Appendix A.2.

\section{Experiments}

\subsection{Datasets}

We conduct experiments with a wide range of extractive QA datasets. Statistics are summarized in Table 3.

MRQA (Fisch et al., 2019). A single-hop extractive QA benchmark that unifies various existing QA datasets into the same format. Here we use the in-domain subset that contains 6 datasets: SQuAD (Rajpurkar et al., 2016), NewsQA (Trischler et al., 2017), TriviaQA (Joshi et al., 2017), SearchQA (Dunn et al., 2017), HotpotQA (Yang et al., 2018) and Natural Questions (Kwiatkowski et al., 2019). Similar to Ram et al. (2021), we adapt these 


\begin{tabular}{|c|c|c|c|c|c|c|c|c|}
\hline Train. Size & Model & SQuAD & TriviaQA & NQ & NewsQA & SearchQA & HotpotQA & Average \\
\hline \multirow{8}{*}{16} & BERT & $9.9 \pm 0.6$ & $15.4 \pm 1.3$ & $20.5 \pm 1.5$ & $6.5 \pm 1.2$ & $16.8 \pm 1.2$ & $9.6 \pm 1.6$ & 13.1 \\
\hline & RoBERTa & $10.3 \pm 1.1$ & $21.0 \pm 3.1$ & $22.5 \pm 2.1$ & $6.7 \pm 2.0$ & $23.4 \pm 3.5$ & $11.2 \pm 1.0$ & 15.9 \\
\hline & SpanBERT & $15.7 \pm 3.6$ & $27.4 \pm 4.1$ & $24.3 \pm 2.1$ & $8.1 \pm 1.4$ & $24.1 \pm 3.2$ & $16.3 \pm 2.0$ & 19.3 \\
\hline & SSPT & $10.8 \pm 1.2$ & $21.2 \pm 3.8$ & $23.7 \pm 4.1$ & $6.5 \pm 1.9$ & $25.8 \pm 2.6$ & $9.1 \pm 1.5$ & 16.2 \\
\hline & Splinter & $16.7 \pm 5.9$ & $23.9_{ \pm 3.8}$ & $25.1 \pm 2.8$ & $11.6 \pm 1.0$ & $23.6 \pm 4.5$ & $15.1 \pm 3.5$ & 19.3 \\
\hline & Splinter* & 54.6 & 18.9 & 27.4 & 20.8 & 26.3 & 24.0 & 28.7 \\
\hline & ReasonBERT & $33.2 \pm 4.0$ & $\underline{37.2} \pm 2.6$ & $\underline{33.1} \pm 2.7$ & $11.8 \pm 2.3$ & $\mathbf{4 6 . 1} \pm 5.2$ & $22 . \overline{4_{ \pm}} 2.8$ & $\underline{30.6}$ \\
\hline & ReasonBERT $_{R}$ & $\underline{41.3} \pm 5.5$ & $\mathbf{4 5 . 5} \pm 5.8$ & $\overline{\mathbf{3 3 . 6}} \pm 3.9$ & $\underline{16.2} \pm 3.2$ & $\underline{45.8} \pm 4.5$ & $\mathbf{3 4 . 1} \pm 2.9$ & $\overline{36.1}$ \\
\hline \multirow{8}{*}{128} & BERT & $21.5 \pm 1.4$ & $23.9 \pm 0.8$ & $31.7 \pm 0.8$ & $11.3 \pm 1.3$ & $32.6 \pm 2.3$ & $14.0 \pm 0.8$ & 22.5 \\
\hline & RoBERTa & $48.8 \pm 4.2$ & $36.0 \pm 2.9$ & $36.4 \pm 2.0$ & $22.8 \pm 2.4$ & $41.3 \pm 2.0$ & $35.2 \pm 1.4$ & 36.7 \\
\hline & SpanBERT & $61.2 \pm 4.7$ & $48.8 \pm 6.6$ & $38.8 \pm 2.6$ & $31.0_{ \pm 5.3}$ & $50.0 \pm 3.7$ & $44.0 \pm 2.3$ & 45.7 \\
\hline & SSPT & $41.5_{ \pm 5.0}$ & $30.3 \pm 3.7$ & $35.0 \pm 2.4$ & $14.0_{ \pm 3.6}$ & $42.8 \pm 3.5$ & $23.7 \pm 3.4$ & 31.2 \\
\hline & Splinter & $55.0 \pm 10.3$ & $45.7 \pm 4.1$ & $41.1 \pm 2.7$ & $33.9_{ \pm 2.8}$ & $48.8 \pm 3.7$ & $46.9_{ \pm 7.1}$ & 45.2 \\
\hline & Splinter* & 72.7 & 44.7 & 46.3 & 43.5 & 47.2 & 54.7 & $\underline{51.5}$ \\
\hline & ReasonBERT & $58.5 \pm 2.2$ & $\underline{56.2} \pm 0.6$ & $\underline{46.7} \pm 2.6$ & $27.8 \pm 0.6$ & $\underline{60.8} \pm 1.7$ & $4 \overline{5.2 \pm 2.3}$ & 49.2 \\
\hline & ReasonBERT $_{\mathrm{R}}$ & $\underline{66.7} \pm 2.9$ & $\overline{\mathbf{6 2 . 1}}_{ \pm 0.9}$ & $\overline{\mathbf{4 9 . 8}}_{ \pm 1.6}$ & $\underline{35.7} \pm 1.5$ & $\overline{\mathbf{6 2 . 3}} \pm 1.7$ & $\mathbf{5 7 . 2} \pm 0.6$ & 55.6 \\
\hline \multirow{8}{*}{1024} & BERT & $64.1 \pm 0.9$ & $41.6 \pm 2.6$ & $50.1 \pm 0.6$ & $43.0_{ \pm 0.3}$ & $53.1 \pm 1.0$ & $46.5 \pm 1.9$ & 49.7 \\
\hline & RoBERTa & $77.9 \pm 0.5$ & $62.2 \pm 1.3$ & $60.3 \pm 0.6$ & $55.0 \pm 0.5$ & $67.5 \pm 0.8$ & $63.4 \pm 0.8$ & 64.4 \\
\hline & SpanBERT & $\underline{81.1} \pm 0.7$ & $67.0 \pm 1.0$ & $63.2 \pm 0.9$ & $\underline{56.4} \pm 0.4$ & $70.0 \pm 0.8$ & $67.6 \pm 1.1$ & 67.5 \\
\hline & SSPT & $\overline{77.6} \pm 1.4$ & $60.1 \pm 2.0$ & $58.7 \pm 0.7$ & $\overline{52.8}_{ \pm 1.1}$ & $65.9 \pm 0.8$ & $63.3 \pm 1.6$ & 63.1 \\
\hline & Splinter & $79.8 \pm 3.5$ & $67.3 \pm 1.5$ & $63.8 \pm 0.5$ & $54.6 \pm 1.4$ & $68.9 \pm 0.3$ & $68.4 \pm 1.2$ & 67.1 \\
\hline & Splinter* & 82.8 & 64.8 & 65.5 & $\mathbf{5 7 . 3}$ & 67.3 & 70.3 & $\underline{68.0}$ \\
\hline & ReasonBERT & $76.9 \pm 0.5$ & $\underline{67.4} \pm 0.5$ & $63.6 \pm 0.6$ & $52.2 \pm 0.5$ & $\underline{70.6} \pm 0.6$ & $67.8 \pm 0.5$ & $\overline{66.4}$ \\
\hline & ReasonBERTR $_{\mathrm{R}}$ & $79.7 \pm 0.3$ & $\overline{\mathbf{7 0 . 1}} \pm 0.2$ & $\underline{65.0} \pm 0.9$ & $54.7 \pm 0.6$ & $\overline{\mathbf{7 2 . 8}} \pm 0.4$ & $\underline{69.7} \pm 0.6$ & 68.7 \\
\hline \multirow{8}{*}{ All } & BERT & 88.8 & 73.6 & 78.7 & 67.5 & 82.0 & 76.2 & 77.8 \\
\hline & RoBERTa & 92.0 & 78.1 & 80.6 & 71.9 & $\underline{85.2}$ & 79.1 & 81.2 \\
\hline & SpanBERT & 92.5 & 79.9 & 80.7 & 71.1 & $\overline{84.8}$ & 80.7 & 81.6 \\
\hline & SSPT & 91.1 & 77.0 & 80.0 & 69.7 & 83.3 & 79.7 & 80.1 \\
\hline & Splinter & 92.4 & 79.7 & 80.3 & 70.8 & 84.0 & $\underline{80.6}$ & 81.3 \\
\hline & Splinter* & $\overline{92.2}$ & $\overline{76.5}$ & 81.0 & 71.3 & 83.0 & $\overline{80.7}$ & 80.8 \\
\hline & ReasonBERT $_{B}$ & 90.3 & 77.5 & 79.9 & 68.7 & 83.7 & 80.5 & 80.1 \\
\hline & ReasonBERTR $_{\mathrm{R}}$ & 91.4 & 78.9 & $\underline{80.8}$ & $\underline{71.4}$ & 85.3 & $\underline{80.6}$ & $\underline{81.4}$ \\
\hline
\end{tabular}

Table 4: Results on MRQA datasets. Best and Second Best results are highlighted. We report the average F1 score over five runs for each dataset, and the macro-average of the six datasets. Splinter* is the result reported in the original paper, where the authors use a deeper model with additional transformation layers on top of the encoder.

datasets to the few-shot setting by randomly sampling smaller subsets from the original training set for training, and use the original development set for testing.

HotpotQA (Yang et al., 2018). A multi-hop QA dataset that requires reasoning over multiple pieces of evidence. Here we follow the distractor setting, where 10 paragraphs are provided to answer a question while only two of them contain relevant information. We split $10 \%$ of the original trainhard split for development, and use the original development set for testing.

NQTables (Kwiatkowski et al., 2019). A subset of the Natural Questions dataset, where at least one answer to the question is present in a table. We extract 19,013 examples from the original training set (307,373 examples) and split them with a 9:1 ratio for training and development. The test set is then created from the original development split $(7,830$ examples) and contains 1,118 examples. Here we only keep tables from the original Wikipedia article as evidence. Similar subsets are also used in Herzig et al. (2021) and Zayats et al. (2021).

HybridQA (Chen et al., 2020). A multi-hop QA dataset with hybrid contexts. Each example contains a table and several linked paragraphs.

We adopt the evaluation script from MRQA ${ }^{3}$, which evaluates the predicted answer using exact match (EM) and token-level F1 metrics.

\subsection{Baselines}

BERT (Devlin et al., 2019). A deep transformer model pre-trained with masked languge model (MLM) and next sentence prediction objectives.

RoBERTa (Liu et al., 2019b). An optimized version of BERT that is pre-trained with a larger text

\footnotetext{
${ }^{3}$ https://github.com/mrqa/ MRQA-Shared-Task-2019
} 
corpus.

SpanBERT (Joshi et al., 2020). A pre-training method designed to better represent and predict spans of text. It extends BERT by masking contiguous random spans, and training the span boundary representation to predict the entire masked span.

SSPT (Glass et al., 2020). A pre-training method designed to improve question answering by training on cloze-like training instances. Unlike ReasonBERT, SSPT only masks a single span in the query sentence and predicts it based on an evidence paragraph provided by a separate retriever.

Splinter (Ram et al., 2021). A pre-training method optimized for few-shot question answering, where the model is pre-trained by masking and predicting recurring spans in a passage.

TAPAS (Herzig et al., 2020). A pre-training method designed to learn representations for tables. The model is pre-trained with MLM on tables and surrounding texts extracted from Wikipedia.

For fair comparison, in each task, we use the same model architecture with different pre-trained encoders, which is similar to the one used for span reasoning in pre-training. We append the [QUESTION] token to a question and construct the input sequence the same way as in pre-training. We then score all the start, end locations and rank all spans $(s, e)$ (See Eqn. 3 and 4 in Appendix). We use a pre-trained encoder and learn the answer extraction layers (S, E in Eqn. 1) from scratch during fine-tuning.

Unless otherwise stated, we use the pre-trained base version so that all models have similar capacity (110M parameters for ReasonBERTB, $125 \mathrm{M}$ parameters for ReasonBERTR, and $111 \mathrm{M}$ parameters for ReasonBERTT).

\subsection{Few-shot Single-hop Text QA}

We first experiment with the easier, single-hop MRQA benchmark under the few-shot setting to show that our pre-training approach learns general knowledge that can be transferred to downstream QA tasks effectively. Results are shown in Table 4. We can see that ReasonBERT outperforms pretrained language models such as BERT, RoBERTa and SpanBERT by a large margin on all datasets, particularly with an average absolute gain of $20.3 \%$ and $14.5 \%$ over BERT and RoBERTa respectively. Compared with pre-training methods such as SSPT and Splinter, ReasonBERT also shows superior performance and obtains the best results on average.

\begin{tabular}{lcccccc}
\hline \multirow{2}{*}{ Model } & \multicolumn{2}{c}{ Recall } & \multicolumn{2}{c}{$1 \%$} & \multicolumn{2}{c}{ Full } \\
& Top 2 & Top 3 & F1 & EM & F1 & EM \\
\hline HGNRoBERTa-Large & - & - & - & - & 82.2 & - \\
HGNBERT & - & - & - & - & 74.8 & - \\
BERT & 92.4 & 96.9 & 39.8 & 28.6 & 71.9 & 57.9 \\
RoBERTa & 93.1 & 97.5 & 56.0 & 43.1 & 76.3 & 62.9 \\
SpanBERT & 93.6 & 97.7 & 56.5 & 44.1 & 76.3 & 62.9 \\
SSPT & 93.9 & 97.9 & 54.7 & 41.8 & 75.4 & 61.5 \\
Splinter & 94.1 & 97.9 & 57.0 & 44.2 & 76.5 & 62.5 \\
ReasonBERTB & 93.8 & 97.8 & 57.6 & 45.3 & 77.2 & 63.4 \\
ReasonBERTR & $\mathbf{9 4 . 0}$ & $\mathbf{9 8 . 0}$ & $\mathbf{6 3 . 1}$ & $\mathbf{5 0 . 2}$ & $\mathbf{7 8 . 1}$ & $\mathbf{6 4 . 8}$ \\
\hline
\end{tabular}

Table 5: Results on HotpotQA.

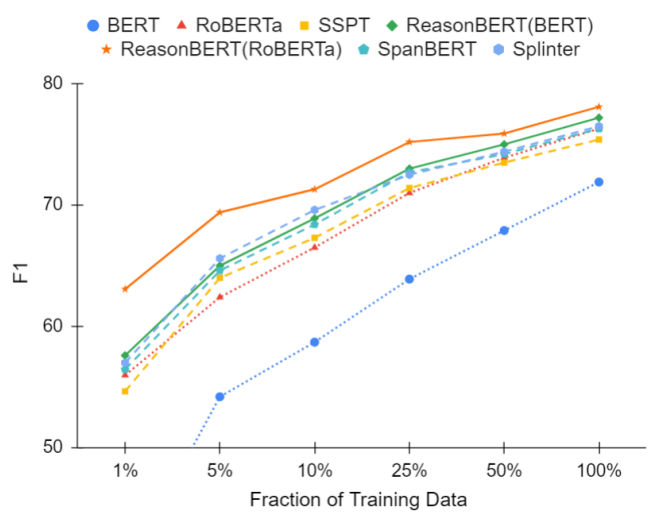

Figure 2: Few-shot learning results on HotpotQA.

Under the full-data setting, ReasonBERT performs competitively and all methods achieve similarly high accuracy. We still demonstrate improvements upon BERT and RoBERTa, and ReasonBERTR second best average score.

\subsection{Multi-hop Text QA}

To demonstrate that our approach is useful in conducting deep reasoning over multiple contexts, we experiment with the HotpotQA dataset. Here we design a simplified multi-hop QA model that first selects relevant paragraphs as evidence, and then extracts the answer from the top selected evidence. Please see Appendix A.3 for implementation details. In addition to comparing ReasonBERT with other pre-training methods using the same base model, we also show results for HGN (Fang et al., 2020), which is one of the top ranked models on the HotpotQA leaderboard that uses a more sophisticated model design.

Results are shown in Table 5. All models perform very well for evidence selection, with over $96 \%$ top 3 recall, but ReasonBERT still maintains a slim lead over baselines. ReasonBERT provides a $5.3 \%$ improvement for BERT and a 1.8\% improvement for RoBERTa on overall F1 score, and outperforms all other pre-training methods. ReasonBERT also outperforms the HGN model with BERT, but 


\begin{tabular}{lcccc}
\hline \multirow{2}{*}{ Model } & \multicolumn{2}{c}{ Dev } & \multicolumn{2}{c}{ Test } \\
& F1 & EM & F1 & EM \\
\hline RoBERTa & 58.9 & 52.8 & 63.6 & 58.1 \\
ReasonBERTR & 61.9 & 56.4 & 66.3 & 60.9 \\
TAPAS & 64.9 & 57.8 & 65.9 & 59.6 \\
ReasonBERT & $\mathbf{6 9 . 2}$ & $\mathbf{6 3 . 5}$ & $\mathbf{7 2 . 5}$ & $\mathbf{6 7 . 3}$ \\
\hline
\end{tabular}

Table 6: Results on NQTables.

is lower than the one using RoBERTa-Large, which is probably due to simpler design and smaller size of the model. We further experiment under the few-shot setting. Here we focus on the QA performance, so we reuse the evidence selector trained with full data for each model, and train the QA module with different fractions of training data. We can see that the advantage of using ReasonBERT is more obvious with limited training data. With $1 \%$ of training data, ReasonBERTR obtains F1 score of $63.1 \%$, a $7.1 \%$ absolute gain over RoBERTa. Results for training the QA model with different fraction of training data is shown in Figure 2. We can see that ReasonBERT obtains larger gain under the few-shot setting.

\subsection{Table QA}

We demonstrate our approach also works with structured data such as tables using the NQTables dataset. We first use a text based RoBERTa encoder as baseline, which linearizes a table as a text sequence, by concatenating tokens row by row and separating cells with the [SEP] token. We then experiment with the structure-aware encoder from TAPAS and compare the pre-trained TAPAS encoder with the one pre-trained using ReasonBERT. Results are shown in Table 6. First, we can see that TAPAS outperforms RoBERTa by $2.3 \%$, demonstrating the importance of modeling the table structure. ReasonBERTR slightly outperforms TAPAS on test set, but ReasonBERT further boosts $\mathrm{F} 1$ to $72.5 \%$, resulting in at least $6.6 \%$ absolute gains over existing methods. Results for training the Table QA model with different fractions of training data are shown in Figure 3. ReasonBERT consistently outperforms TAPAS while ReasonBERTR gradually matches the performance of TAPAS with the increasing of training data.

\subsection{Hybrid QA}

We further evaluate our approach on HybridQA, a multi-hop question answering dataset using both text and tables as evidence. Chen et al. (2020) proposes a baseline model HYBRIDER that divides

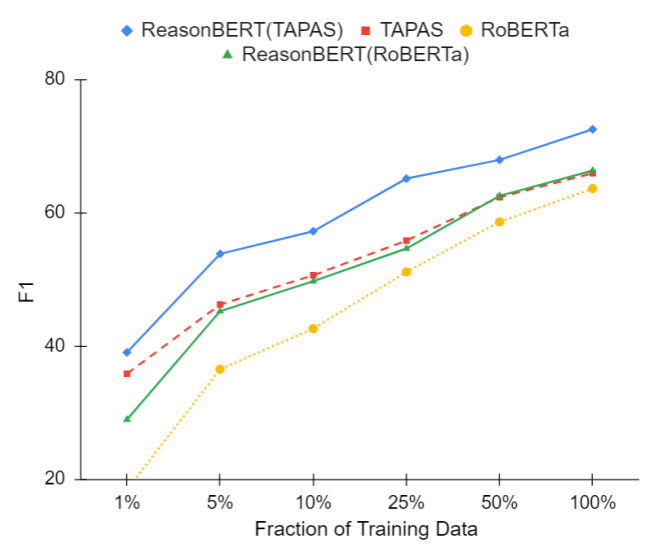

Figure 3: Few-shot learning results on NQTables.

\begin{tabular}{lcccccc}
\hline \multirow{2}{*}{ Model } & \multicolumn{2}{c}{ Cell Selection } & \multicolumn{2}{c}{ Dev } & \multicolumn{2}{c}{ Test } \\
& Top 1 & Top 2 & F1 & EM & F1 & EM \\
\hline HYBRIDERBERT-Base & - & - & 50.9 & 43.7 & 50.2 & 42.5 \\
HYBRIDERBERT-Large & 68.5 & - & 50.7 & 44.0 & 50.6 & 43.8 \\
TAPAS+RoBERTa & 73.3 & 79.7 & 64.0 & 57.3 & 63.3 & 56.1 \\
ReasonBERT & $\mathbf{7 6 . 1}$ & $\mathbf{8 1 . 3}$ & $\mathbf{6 7 . 2}$ & $\mathbf{6 0 . 3}$ & $\mathbf{6 5 . 3}$ & $\mathbf{5 8 . 0}$ \\
\hline
\end{tabular}

Table 7: Results on HybridQA.

the problem into four tasks: linking, ranking, hopping and reading comprehension. We follow their design but simplify the model by merging ranking and hopping into a single cell selection task. We use the linking results from Chen et al. (2020), and then train a table based cell selector to select the cell which is the answer or is linked to the passage that contains the answer. Finally, we train a text based QA model to extract the final answer by taking the table snippet that contains the selected cell, and concatenating it with the hyperlinked passage as evidence. Please see Appendix A.3 for implementation details. Results are shown in Table 7. First, we can see that our simplified architecture works surprisingly well, with TAPAS for cell selection and RoBERTa for QA, we already outperform HYBRIDER. The performance is further improved by replacing the encoders with ReasonBERT $\mathrm{T}$ and ReasonBERT $_{R}$, and substantially outperforms the best model on the leaderboard (52.04 EM) at the time of submission.

\section{Ablation Study}

We further conduct ablation studies on HotpotQA to verify our design choices, summarized in Table 8 . Here we remove different components of ReasonBERTR and test them under both the fulldata and few-shot setting (with 1024 examples). To save computing resources, here all models are pre-trained with 5 epochs. We can see that combining multiple pieces of evidence and predicting 


\begin{tabular}{lcccc}
\hline \multirow{2}{*}{ Model } & \multicolumn{2}{c}{1024} & \multicolumn{2}{c}{ Full } \\
& F1 & EM & F1 & EM \\
\hline ReasonBERTR & 65.2 & 52.8 & 79.2 & 65.8 \\
- MLM & 63.7 & 51.3 & 77.7 & 64.0 \\
- Unanswerable Ent. & 64.4 & 51.8 & 78.4 & 65.0 \\
- Multiple Evidences & 60.8 & 48.6 & 77.8 & 64.5 \\
\hline
\end{tabular}

Table 8: Ablation study on HotpotQA.

multiple masked spans simultaneously brings the most gain, especially under the few-shot setting. This is probably because the setting allows us to simulate complex reasoning chains and encourage the model to do deep reasoning. Masking unanswerable entities and utilizing MLM also help to improve performance.

\section{Related Work}

Language Model Pre-training. Contextualized word representations pre-trained on large-scale unlabeled text corpus have been widely used in NLP lately. Most prevalent approaches are variants of pre-trained language models such as BERT (Devlin et al., 2019) and RoBERTa (Liu et al., 2019b). More recently, self-supervised pre-training has also shown promising results on modalities other than plain text, such as tables (Herzig et al., 2020; Deng et al., 2020; Iida et al., 2021), knowledge bases (Zhang et al., 2019; Peters et al., 2019) and image-text (Su et al., 2020). Meanwhile, there has also been work that uses pre-training to accommodate specific needs of downstream NLP tasks, such as open-domain retrieval (Guu et al., 2020), representing and predicting spans of text (Joshi et al., 2020) and semantic parsing (Yu et al., 2020; Deng et al., 2021).

Machine Reading Comprehension. Machine reading comprehension (MRC) or extractive QA has become an important testbed for natural language understanding evaluation (Fisch et al., 2019). The conventional method to train an MRC model usually relies on large-scale supervised training data (Chen et al., 2017; Zhang et al., 2020). Recently, more and more work has focused on developing self-supervised methods that can reduce the need for labeled data for more efficient domain adaptation, while achieving the same or even better performance. One direction is question generation (Pan et al., 2021), which automatically generates questions and answers from unstructured and structured data sources using rules or neural generators. Recent work also tries to directly simulate questions with cloze-like query sentences. Splin- ter (Ram et al., 2021) proposes to pre-train the model by masking and predicting recurring spans. However, this limits the query and context to come from the same passage. In contrast, SSPT (Glass et al., 2020) also pre-trains with a span selection objective, but uses a separate document retriever to get relevant paragraphs as context.

Our work is most related to SSPT, but uses distant supervision to collect query-evidence pairs and thus obviate the need for a retriever. Meanwhile, to encourage the model to learn complex reasoning, we mimic different types of reasoning chains by masking multiple entities, including unanswerable ones, and simultaneously inferring them from disjoint pieces of evidence. Our method also works with heterogeneous sources including both text and tables, while most existing work considers only text-based question answering.

\section{Conclusion and Future Work}

We propose ReasonBERT, a novel pre-training method to enhance the reasoning ability of language models. The resulting model obtains substantial improvements on multi-hop and hybrid QA tasks that require complex reasoning, and demonstrates superior few-shot performance. In the future, we plan to use our query-evidence pairs collected by distant supervision to improve the retrieval performance for open-domain QA, as well as empower ReasonBERT to handle more types of reasoning, like comparison and numeric reasoning, in natural language understanding.

\section{Acknowledgements}

We would like to thank the anonymous reviewers for their helpful comments. Authors at The Ohio State University were sponsored in part by Google Faculty Award, the Army Research Office under cooperative agreements W911NF-17-1-0412, NSF Grant IIS1815674, NSF CAREER \#1942980, Fujitsu gift grant, and Ohio Supercomputer Center (Center, 1987). The views and conclusions contained herein are those of the authors and should not be interpreted as representing the official policies, either expressed or implied, of the Army Research Office or the U.S. Government. The U.S. Government is authorized to reproduce and distribute reprints for Government purposes notwithstanding any copyright notice herein. Research was also supported with Cloud TPUs from Google's TPU Research Cloud (TRC). 


\section{References}

Jacob Andreas, John Bufe, David Burkett, Charles Chen, Josh Clausman, Jean Crawford, Kate Crim, Jordan DeLoach, Leah Dorner, Jason Eisner, Hao Fang, Alan Guo, David Hall, Kristin Hayes, Kellie Hill, Diana Ho, Wendy Iwaszuk, Smriti Jha, Dan Klein, Jayant Krishnamurthy, Theo Lanman, Percy Liang, Christopher H. Lin, Ilya Lintsbakh, Andy McGovern, Aleksandr Nisnevich, Adam Pauls, Dmitrij Petters, Brent Read, Dan Roth, Subhro Roy, Jesse Rusak, Beth Short, Div Slomin, Ben Snyder, Stephon Striplin, Yu Su, Zachary Tellman, Sam Thomson, Andrei Vorobev, Izabela Witoszko, Jason Wolfe, Abby Wray, Yuchen Zhang, and Alexander Zotov. 2020. Task-oriented dialogue as dataflow synthesis. Transactions of the Association for Computational Linguistics, 8:556-571.

Abhijeet Awasthi, Sunita Sarawagi, Rasna Goyal, Sabyasachi Ghosh, and Vihari Piratla. 2019. Parallel iterative edit models for local sequence transduction. In Proceedings of the 2019 Conference on Empirical Methods in Natural Language Processing and the 9th International Joint Conference on Natural Language Processing (EMNLP-IJCNLP), pages 42604270, Hong Kong, China. Association for Computational Linguistics.

Tom B. Brown, Benjamin Mann, Nick Ryder, Melanie Subbiah, Jared Kaplan, Prafulla Dhariwal, Arvind Neelakantan, Pranav Shyam, Girish Sastry, Amanda Askell, Sandhini Agarwal, Ariel Herbert-Voss, Gretchen Krueger, Tom Henighan, Rewon Child, Aditya Ramesh, Daniel M. Ziegler, Jeffrey Wu, Clemens Winter, Christopher Hesse, Mark Chen, Eric Sigler, Mateusz Litwin, Scott Gray, Benjamin Chess, Jack Clark, Christopher Berner, Sam McCandlish, Alec Radford, Ilya Sutskever, and Dario Amodei. 2020. Language models are few-shot learners. In Advances in Neural Information Processing Systems 33: Annual Conference on Neural Information Processing Systems 2020, NeurIPS 2020, December 6-12, 2020, virtual.

Ohio Supercomputer Center. 1987. Ohio supercomputer center.

Danqi Chen, Adam Fisch, Jason Weston, and Antoine Bordes. 2017. Reading Wikipedia to answer opendomain questions. In Proceedings of the 55th Annual Meeting of the Association for Computational Linguistics (Volume 1: Long Papers), pages 18701879, Vancouver, Canada. Association for Computational Linguistics.

Wenhu Chen, Hanwen Zha, Zhiyu Chen, Wenhan Xiong, Hong Wang, and William Yang Wang. 2020. HybridQA: A dataset of multi-hop question answering over tabular and textual data. In Findings of the Association for Computational Linguistics: EMNLP 2020, pages 1026-1036, Online. Association for Computational Linguistics.

Xiang Deng, Ahmed Hassan Awadallah, Christopher Meek, Oleksandr Polozov, Huan Sun, and Matthew
Richardson. 2021. Structure-grounded pretraining for text-to-SQL. In Proceedings of the 2021 Conference of the North American Chapter of the Association for Computational Linguistics: Human Language Technologies, pages 1337-1350, Online. Association for Computational Linguistics.

Xiang Deng, Huan Sun, Alyssa Lees, You Wu, and Cong Yu. 2020. Turl: table understanding through representation learning. Proceedings of the VLDB Endowment, 14(3):307-319.

Jacob Devlin, Ming-Wei Chang, Kenton Lee, and Kristina Toutanova. 2019. BERT: Pre-training of deep bidirectional transformers for language understanding. In Proceedings of the 2019 Conference of the North American Chapter of the Association for Computational Linguistics: Human Language Technologies, Volume 1 (Long and Short Papers), pages 4171-4186, Minneapolis, Minnesota. Association for Computational Linguistics.

Matthew Dunn, Levent Sagun, Mike Higgins, V. U. Güney, Volkan Cirik, and Kyunghyun Cho. 2017. Searchqa: A new q\&a dataset augmented with context from a search engine. ArXiv, abs/1704.05179.

Yuwei Fang, Siqi Sun, Zhe Gan, Rohit Pillai, Shuohang Wang, and Jingjing Liu. 2020. Hierarchical graph network for multi-hop question answering. In Proceedings of the 2020 Conference on Empirical Methods in Natural Language Processing (EMNLP), pages 8823-8838, Online. Association for Computational Linguistics.

Adam Fisch, Alon Talmor, Robin Jia, Minjoon Seo, Eunsol Choi, and Danqi Chen. 2019. MRQA 2019 shared task: Evaluating generalization in reading comprehension. In Proceedings of the 2nd Workshop on Machine Reading for Question Answering, pages 1-13, Hong Kong, China. Association for Computational Linguistics.

Michael Glass, Alfio Gliozzo, Rishav Chakravarti, Anthony Ferritto, Lin Pan, G P Shrivatsa Bhargav, Dinesh Garg, and Avi Sil. 2020. Span selection pretraining for question answering. In Proceedings of the 58th Annual Meeting of the Association for Computational Linguistics, pages 2773-2782, Online. Association for Computational Linguistics.

Kelvin Guu, Kenton Lee, Zora Tung, Panupong Pasupat, and Ming-Wei Chang. 2020. REALM: Retrieval-augmented language model pre-training. arXiv preprint arXiv:2002.08909.

Jonathan Herzig, Thomas Müller, Syrine Krichene, and Julian Eisenschlos. 2021. Open domain question answering over tables via dense retrieval. In Proceedings of the 2021 Conference of the North American Chapter of the Association for Computational Linguistics: Human Language Technologies, pages 512-519, Online. Association for Computational Linguistics. 
Jonathan Herzig, Pawel Krzysztof Nowak, Thomas Müller, Francesco Piccinno, and Julian Eisenschlos. 2020. TaPas: Weakly supervised table parsing via pre-training. In Proceedings of the 58th Annual Meeting of the Association for Computational Linguistics, pages 4320-4333, Online. Association for Computational Linguistics.

Hiroshi Iida, Dung Thai, Varun Manjunatha, and Mohit Iyyer. 2021. TABBIE: Pretrained representations of tabular data. In Proceedings of the 2021 Conference of the North American Chapter of the Association for Computational Linguistics: Human Language Technologies, pages 3446-3456, Online. Association for Computational Linguistics.

Yichen Jiang, Shikha Bordia, Zheng Zhong, Charles Dognin, Maneesh Singh, and Mohit Bansal. 2020. HoVer: A dataset for many-hop fact extraction and claim verification. In Findings of the Association for Computational Linguistics: EMNLP 2020, pages 3441-3460, Online. Association for Computational Linguistics.

Mandar Joshi, Danqi Chen, Yinhan Liu, Daniel S. Weld, Luke Zettlemoyer, and Omer Levy. 2020. SpanBERT: Improving pre-training by representing and predicting spans. Transactions of the Association for Computational Linguistics, 8:64-77.

Mandar Joshi, Eunsol Choi, Daniel Weld, and Luke Zettlemoyer. 2017. TriviaQA: A large scale distantly supervised challenge dataset for reading comprehension. In Proceedings of the 55th Annual Meeting of the Association for Computational Linguistics (Volume 1: Long Papers), pages 1601-1611, Vancouver, Canada. Association for Computational Linguistics.

Tom Kwiatkowski, Jennimaria Palomaki, Olivia Redfield, Michael Collins, Ankur Parikh, Chris Alberti, Danielle Epstein, Illia Polosukhin, Jacob Devlin, Kenton Lee, Kristina Toutanova, Llion Jones, Matthew Kelcey, Ming-Wei Chang, Andrew M. Dai, Jakob Uszkoreit, Quoc Le, and Slav Petrov. 2019. Natural questions: A benchmark for question answering research. Transactions of the Association for Computational Linguistics, 7:452-466.

Xiaodong Liu, Pengcheng He, Weizhu Chen, and Jianfeng Gao. 2019a. Multi-task deep neural networks for natural language understanding. In Proceedings of the 57th Annual Meeting of the Association for Computational Linguistics, pages 4487-4496, Florence, Italy. Association for Computational Linguistics.

Y. Liu, Myle Ott, Naman Goyal, Jingfei Du, Mandar Joshi, Danqi Chen, Omer Levy, M. Lewis, Luke Zettlemoyer, and Veselin Stoyanov. 2019b. Roberta: A robustly optimized bert pretraining approach. ArXiv, abs/1907.11692.

Ilya Loshchilov and Frank Hutter. 2019. Decoupled weight decay regularization. In 7 th International Conference on Learning Representations,
ICLR 2019, New Orleans, LA, USA, May 6-9, 2019. OpenReview.net.

Mike Mintz, Steven Bills, Rion Snow, and Daniel Jurafsky. 2009a. Distant supervision for relation extraction without labeled data. In Proceedings of the Joint Conference of the 47th Annual Meeting of the ACL and the 4th International Joint Conference on Natural Language Processing of the AFNLP, pages 1003-1011, Suntec, Singapore. Association for Computational Linguistics.

Mike Mintz, Steven Bills, Rion Snow, and Daniel Jurafsky. 2009b. Distant supervision for relation extraction without labeled data. In Proceedings of the Joint Conference of the 47th Annual Meeting of the ACL and the 4th International Joint Conference on Natural Language Processing of the AFNLP, pages 1003-1011, Suntec, Singapore. Association for Computational Linguistics.

Liangming Pan, Wenhu Chen, Wenhan Xiong, MinYen Kan, and William Yang Wang. 2021. Unsupervised multi-hop question answering by question generation. In Proceedings of the 2021 Conference of the North American Chapter of the Association for Computational Linguistics: Human Language Technologies, pages 5866-5880, Online. Association for Computational Linguistics.

Matthew E. Peters, Mark Neumann, Robert Logan, Roy Schwartz, Vidur Joshi, Sameer Singh, and Noah A Smith. 2019. Knowledge enhanced contextual word representations. In Proceedings of the 2019 Conference on Empirical Methods in Natural Language Processing and the 9th International Joint Conference on Natural Language Processing (EMNLPIJCNLP), pages 43-54, Hong Kong, China. Association for Computational Linguistics.

Alec Radford and Karthik Narasimhan. 2018. Improving language understanding by generative pretraining.

Alec Radford, Jeffrey Wu, R. Child, David Luan, Dario Amodei, and Ilya Sutskever. 2019. Language models are unsupervised multitask learners.

Pranav Rajpurkar, Jian Zhang, Konstantin Lopyrev, and Percy Liang. 2016. SQuAD: 100,000+ questions for machine comprehension of text. In Proceedings of the 2016 Conference on Empirical Methods in Natural Language Processing, pages 2383-2392, Austin, Texas. Association for Computational Linguistics.

Ori Ram, Yuval Kirstain, Jonathan Berant, Amir Globerson, and Omer Levy. 2021. Few-shot question answering by pretraining span selection. In Proceedings of the 59th Annual Meeting of the Association for Computational Linguistics and the 11th International Joint Conference on Natural Language Processing (Volume 1: Long Papers), pages 30663079, Online. Association for Computational Linguistics. 
Subendhu Rongali, Luca Soldaini, Emilio Monti, and Wael Hamza. 2020. Don't parse, generate! A sequence to sequence architecture for task-oriented semantic parsing. In $W W W$ '20: The Web Conference 2020, Taipei, Taiwan, April 20-24, 2020, pages 2962-2968. ACM / IW3C2.

Weijie Su, Xizhou Zhu, Yue Cao, Bin Li, Lewei Lu, Furu Wei, and Jifeng Dai. 2020. VL-BERT: pretraining of generic visual-linguistic representations. In 8th International Conference on Learning Representations, ICLR 2020, Addis Ababa, Ethiopia, April 26-30, 2020. OpenReview.net.

Adam Trischler, Tong Wang, Xingdi Yuan, Justin Harris, Alessandro Sordoni, Philip Bachman, and Kaheer Suleman. 2017. NewsQA: A machine comprehension dataset. In Proceedings of the 2nd Workshop on Representation Learning for NLP, pages 191-200, Vancouver, Canada. Association for Computational Linguistics.

Alex Wang, Yada Pruksachatkun, Nikita Nangia, Amanpreet Singh, Julian Michael, Felix Hill, Omer Levy, and Samuel R. Bowman. 2019a. Superglue: A stickier benchmark for general-purpose language understanding systems. In Advances in Neural Information Processing Systems 32: Annual Conference on Neural Information Processing Systems 2019, NeurIPS 2019, December 8-14, 2019, Vancouver, BC, Canada, pages 3261-3275.

Alex Wang, Amanpreet Singh, Julian Michael, Felix Hill, Omer Levy, and Samuel R. Bowman. 2019b. GLUE: A multi-task benchmark and analysis platform for natural language understanding. In $7 \mathrm{th}$ International Conference on Learning Representations, ICLR 2019, New Orleans, LA, USA, May 6-9, 2019. OpenReview.net.

Johannes Welbl, Pontus Stenetorp, and Sebastian Riedel. 2018. Constructing datasets for multi-hop reading comprehension across documents. Transactions of the Association for Computational Linguistics, 6:287-302.

Thomas Wolf, Lysandre Debut, Victor Sanh, Julien Chaumond, Clement Delangue, Anthony Moi, Pierric Cistac, Tim Rault, Remi Louf, Morgan Funtowicz, Joe Davison, Sam Shleifer, Patrick von Platen, Clara Ma, Yacine Jernite, Julien Plu, Canwen Xu, Teven Le Scao, Sylvain Gugger, Mariama Drame, Quentin Lhoest, and Alexander Rush. 2020. Transformers: State-of-the-art natural language processing. In Proceedings of the 2020 Conference on Empirical Methods in Natural Language Processing. System Demonstrations, pages 38-45, Online. Association for Computational Linguistics.

Hu Xu, Bing Liu, Lei Shu, and Philip Yu. 2019. BERT post-training for review reading comprehension and aspect-based sentiment analysis. In Proceedings of the 2019 Conference of the North American Chapter of the Association for Computational Linguistics. Human Language Technologies, Volume 1 (Long and Short Papers), pages 2324-2335, Minneapolis, Minnesota. Association for Computational Linguistics.

Zhilin Yang, Peng Qi, Saizheng Zhang, Yoshua Bengio, William Cohen, Ruslan Salakhutdinov, and Christopher D. Manning. 2018. HotpotQA: A dataset for diverse, explainable multi-hop question answering. In Proceedings of the 2018 Conference on Empirical Methods in Natural Language Processing, pages 2369-2380, Brussels, Belgium. Association for Computational Linguistics.

Tao Yu, Chien-Sheng Wu, Xi Victoria Lin, Yi Chern Tan, Xinyi Yang, Dragomir Radev, Caiming Xiong, et al. 2020. Grappa: Grammar-augmented pretraining for table semantic parsing. In International Conference on Learning Representations.

Vicky Zayats, Kristina Toutanova, and Mari Ostendorf. 2021. Representations for question answering from documents with tables and text. In Proceedings of the 16th Conference of the European Chapter of the Association for Computational Linguistics: Main Volume, pages 2895-2906, Online. Association for Computational Linguistics.

Zhengyan Zhang, Xu Han, Zhiyuan Liu, Xin Jiang, Maosong Sun, and Qun Liu. 2019. ERNIE: Enhanced language representation with informative entities. In Proceedings of the 57th Annual Meeting of the Association for Computational Linguistics, pages 1441-1451, Florence, Italy. Association for Computational Linguistics.

Zhuosheng Zhang, J. Yang, and Hai Zhao. 2020. Retrospective reader for machine reading comprehension. ArXiv, abs/2001.09694. 


\section{A Implementation Details}

\section{A.1 Pre-training Data Details}

We extract paragraphs from Wikipedia XML dump $^{4}$ use JWPL ${ }^{5}$ and tables use wikitextparser ${ }^{6}$. The paragraphs are then processed with SparkNLP ${ }^{7}$ for sentence boundary detection and named entity recognition.

Table 9 and Table 10 show some examples of the query-evidence pairs we collected for pre-training. The selected entities are underlined. During pretraining, we will mask some of the entities in the query and recover them based on the evidence. As the pre-training data is collected via distant supervision, it contains some noise. Here we also include some bad examples where the evidence does not express the same relation between the selected entities as the query sentence (highlighted in red).

\section{A.2 Pre-training Details}

We set the max length of query sentences to 100 tokens and the max length of single piece of evidence to 200 if there are two evidence selections or 400 if there is only one. For textual evidence, we include the neighbouring sentences from the same paragraph as extra context for the selected evidence sentence and clip to the max evidence length. For tabular evidence, we take a snippet of the original table, and truncate the cells to 20 tokens. We always keep the first row and column in the table, as they often contain important information such as headers and subject entities. Based on the selected entity pair, we sample up to 5 columns and include as many rows as possible until reaching the budget.

We initialize our encoder with BERT-Base ${ }^{8}$ and RoBERTa-Base ${ }^{9}$ for the text part, and TAPASbase $^{10}$ for the table part. We train ReasonBERT using AdamW (Loshchilov and Hutter, 2019) for 10 epochs with batches of 256 sequences of length 512 ; this is approximately $290 \mathrm{k}$ steps with textonly data, and $120 \mathrm{k}$ steps with hybrid data. We base our implementation on Huggingface Transformers (Wolf et al., 2020), and train on a single

\footnotetext{
${ }^{4}$ https: / / dumps.wikimedia.org/

${ }^{5}$ https://dkpro.github.io/dkpro-jwpl/

${ }^{6}$ https://github.com/5j9/wikitextparser

${ }^{7}$ https://nlp. johnsnowlabs.com/

${ }^{8}$ https://huggingface.co/

bert-base-uncased/tree/main

${ }^{9}$ https://huggingface.co/roberta-base/ tree/main

${ }^{10}$ https: / / huggingface.co/google/ tapas-base/tree/no_reset
}

eight-core TPU on the Google Cloud Platform.

\section{A.3 Fine-tuning Details}

To extract the answer span from given evidence, we score all the start, end locations and rank all spans $(s, e)$ by $g(s, e \mid q, E)$ as follows:

$$
\begin{aligned}
f_{\text {start }}=\mathbf{x}_{s}^{\top} \mathbf{S} \mathbf{x}_{q}, & f_{\text {end }}=\mathbf{x}_{e}^{\top} \mathbf{E} \mathbf{x}_{q} \\
g(s, e \mid q, E) & =f_{\text {start }}(s \mid q, E) \\
& +f_{\text {end }}(e \mid q, E) \\
& -f_{\text {start }}([C L S] \mid q, E) \\
& -f_{\text {end }}([\mathrm{CLS}] \mid q, E)
\end{aligned}
$$

For all fine-tuning experiments, we set the batch size to 20 and use a maximal learning rate of 5 . $10^{-5}$, which warms up in the first $10 \%$ of the steps, and then decays linearly. We use the development set for model selection if it is present, otherwise we use the last model checkpoint.

Single-hop text QA. We split the text sequence to fit the max input length by sliding a window with a stride of 128 tokens.

For the few-shot setting, we fine-tune the model for either 10 epochs or 200 steps (whichever is larger). For the fully supervised setting, we finetune the model for 2 epochs.

Multi-hop text QA. We design a simplified multihop QA model that first selects relevant paragraphs as evidence, and then extracts the answer from the selected evidence samples. Specifically, we first generate all possible paragraphs by sliding a 200token window over all articles with a stride of 128 tokens. We then train an evidence selector to pick the top 3 evidence samples. As the information for answering a question in HotpotQA is scattered in two articles, we list all possible combinations of paragraphs that come from two different articles and concatenate them together to form the final evidence. We then use the base QA model to extract the answer based on the question and the combined evidence.

We fine-tune the evidence selector model for 2 epochs, and the QA model for 5 epochs with full data. For the few-shot setting, we fine-tune the QA model for 10 epochs with $1 \&, 5 \%$ and $10 \%$ of the training data, and for 5 epochs with $25 \%$ and $50 \%$ of the training data.

Table QA. For the text based model, We split the text sequence to fit the max input length by sliding a window with a stride of 128 tokens. For the table based model, we truncate each cell to 50 tokens, 
and split the table into snippets horizontally. Same as pre-training, we include the first row and column in each table snippet.

We fine-tune the model for 5 epochs with full data. For the few-shot setting, we fine-tune the QA model for 10 epochs with $1 \&, 5 \%$ and $10 \%$ of the training data, and for 5 epochs with $25 \%$ and $50 \%$ of the training data.

Hybrid QA. Chen et al. (2020) proposes a baseline model that divides the problem into four tasks: 1 ) linking: link questions to their corresponding cells using heuristics. 2) ranking: rank the linked cells use a neural model. 3) hopping: based on the cell selected in the last step, decide which neighboring cell or itself contains the final answer. 4) reading comprehension: extract the answer from the predicted cell or its linked paragraph. We follow their design and simplify the model by merging ranking and hopping into a single cell selection task. We use the linking results from Chen et al. (2020). For each linked cell, we take a snippet out of the original table including the headers, the entire row of the linked cell, and concatenate the evidence sentence to the cell if it is linked through the hyperlinked passage. To select the cell, we train the model to select separately on the token, row and column level, and aggregate the final scores . More specifically, we calculate the probability of selecting on the token and row level as follows:

$$
\begin{gathered}
P(t \mid q, E)=\frac{\exp \left(\mathbf{x}_{t}^{\top} \mathbf{S} \mathbf{x}_{a_{i}}\right)}{\sum_{k} \exp \left(\mathbf{x}_{k}^{\top} \mathbf{S} \mathbf{x}_{a_{i}}\right)} \\
S_{\text {cell }}=\operatorname{mean}_{x_{i} \in \text { cell }}\left(\mathbf{x}_{i}^{\top} \mathbf{R} \mathbf{x}_{a}\right) \\
P\left(r_{a}=j \mid q, E\right)=\frac{\exp \left(\max _{\text {cell } \in r_{j}} S_{\text {cell }}\right)}{\sum_{k} \exp \left(\max _{\text {cell } \in r_{k}} S_{\text {cell }}\right)}
\end{gathered}
$$

Here $\mathbf{S}$ is the weight matrix of the token selection header, we only consider the first token in each cell, and $t$ is the first token of the selected cell. $\mathbf{R}$ is the weight matrix of row selection header, and the column selection probability is calculated similarly with another column selection header. We first score each cell by averaging over all tokens in that cell. We then do a max pooling over all cells in the row or column so the model can focus on the strongest signal, for example the column header. The final probability of selecting a cell is the sum of token, row and column scores.

The input for the QA model then contains the header of the table, the row of the selected cell, and the hyperlinked passage.

We fine-tune the cell selection model for 2 epochs and the QA model for 3 epochs. 


\begin{tabular}{|c|c|}
\hline Query & Evidence \\
\hline $\begin{array}{l}\text { "I Thought I Lost You" was nominated } \\
\text { for Broadcast Film Critics Association } \\
\text { Award for Best Song and Golden Globe } \\
\text { Award for Best Original Song, but lost } \\
\text { both to Bruce Springsteen's } \\
\text { "The Wrestler" from The Wrestler } \\
\text { (2008). }\end{array}$ & $\begin{array}{l}\text { On January 11, 2009, Springsteen won the Golden Globe Award } \\
\text { for Best Song for "The Wrestler", from the Darren Aronofsky } \\
\text { film by the same name. } \\
\text { "I I Thought I Lost You" was nominated for the Broadcast Film } \\
\text { Critics Association Award for Best Song at the 14th Broadcast } \\
\text { Film Critics Association Award, but lost to Bruce Springsteen's } \\
\text { "The Wrestler" from The Wrestler (2008). }\end{array}$ \\
\hline $\begin{array}{l}\text { Film critic Roger Ebert compared it to } \\
\text { John Carpenter's Halloween, noting: } \\
\text { "Blue Steel" is a sophisticated update } \\
\text { of Halloween, the movie that first made } \\
\text { Jamie Lee Curtis a star. }\end{array}$ & $\begin{array}{l}\text { Historian Nicholas Rogers notes that film critics contend that } \\
\text { Carpenter's direction and camera work made Halloween a "re- } \\
\text { sounding success." Roger Ebert remarks, ... } \\
\text { Since Jamie Lee Curtis, the main actress from the original and } \\
\text { the sequel Halloween II (1981), wanted to reunite the cast and } \\
\text { crew of the original film, she asked Carpenter to direct Hal- } \\
\text { loween H20: } 20 \text { Years Later. }\end{array}$ \\
\hline $\begin{array}{l}\text { A hybrid disc is an optical disc that } \\
\text { has multiple file system installed on it, } \\
\text { typically ISO } \underline{9660} \text { and } \mathrm{HFS}+\text { (or } \underline{H F S} \\
\text { on older discs). }\end{array}$ & $\begin{array}{l}\text { Hierarchical File System ( } \underline{H F S} \text { ) is a proprietary file system } \\
\text { developed by Apple Inc. for use in computer systems running } \\
\text { Mac OS. } \\
\text { ISO } 9660 \text { is a file system for optical disc media. Being sold by } \\
\text { the International Organization for Standardization (ISO) the file } \\
\text { system is considered an international technical standard. }\end{array}$ \\
\hline $\begin{array}{l}\text { After } 1709 \text {, the heads of the } \\
\text { House of Orleans branch of } \\
\text { the House of Bourbon ranked as the } \\
\text { prince of the Blood - this meant that } \\
\text { the dukes could be addressed as } \\
\text { Monsieur le Prince (a style they did not, } \\
\text { however, use). }\end{array}$ & $\begin{array}{l}\text { From } \underline{1709} \text { until the French Revolution, the Orleans dukes were } \\
\text { next in the order of succession to the French throne after members } \\
\text { of the senior branch of the House of Bourbon, descended from } \\
\text { Louis XIV. } \\
\text { Restored briefly in } 1814 \text { and definitively in } 1815 \text { after the fall of } \\
\text { the First French Empire, the senior line of the Bourbons was } \\
\text { finally overthrown in the July Revolution of } 1830 \text {. A cadet } \\
\text { Bourbon branch, the House of Orleans, then ruled for } 18 \text { years } \\
(1830-1848) \text {, until it too was overthrown. }\end{array}$ \\
\hline $\begin{array}{l}\text { The Citroen C6 is an executive car } \\
\text { produced by the French car maker } \\
\text { Citroen from } 2005 \text { to } 2012 \text {. }\end{array}$ & $\begin{array}{l}\text { The } \underline{C 6} \text { was aimed as a stylish alternative to executive cars, like } \\
\text { the BMW } 5 \text { Series and the Audi A6, and it has been described } \\
\text { as "spaceship that rides on air", "charmingly idiosyncratic" and } \\
\text { "refreshingly different". } \\
\text { In } 2012 \text {, Citroen announced plans to enter the World Touring } \\
\text { Car Championship. The team transformed a DS3 WRC into a } \\
\text { laboratory vehicle to help with early development, while ... }\end{array}$ \\
\hline $\begin{array}{l}\text { Leaving the Market Street subway at } \\
\text { Ferry Portal heading south, the T Third } \\
\text { Street follows The Embarcadero south } \\
\text { of Market Street, then veers onto King } \\
\text { Street in front of } \text { Oracle Park until it } \\
\text { reaches the Caltrain station terminal. }\end{array}$ & $\begin{array}{l}\text { the 4th \& King Caltrain station is } 1.5 \text { blocks from the stadium, } \\
\text { and the Oracle Park Ferry Terminal is outside the east edge of } \\
\text { the ballpark beyond the center field bleachers. } \\
\text { the southwestern end of the Market Street subway connects to } \\
\text { the much-older Twin Peaks Tunnel, and the northeastern end } \\
\text { connects to surface tracks along the The Embarcadero. }\end{array}$ \\
\hline
\end{tabular}

Table 9: Pre-training data examples, text-only setting. 


\begin{tabular}{|c|c|c|c|}
\hline Query & \multicolumn{3}{|c|}{ Evidence } \\
\hline $\begin{array}{l}\text { Rowland Barran (7 August } 1858-6 \\
\text { August 1949) was an English Liberal } \\
\text { Party politician and } \underline{\text { Member of }}\end{array}$ & \multicolumn{3}{|c|}{$\begin{array}{l}\text { Rowland Barran was the youngest son of Sir John Barran, a } \\
\text { pioneer in clothing manufacture and Member of Parliament for } \\
\text { Leeds and Otley. }\end{array}$} \\
\hline \multirow[t]{2}{*}{ Parliament. } & Year & Member & Party \\
\hline & $\begin{array}{l}1885 \\
1902 \\
1918\end{array}$ & $\begin{array}{l}\text { William Jackson } \\
\text { Rowland Barran } \\
\text { Alexander } \\
\text { Farquharson }\end{array}$ & $\begin{array}{l}\text { Conservative } \\
\text { Liberal } \\
\text { Coalition Liberal }\end{array}$ \\
\hline
\end{tabular}

"It Ain't Over 'til It's Over" is a song recorded, written, and produced by American musician Lenny Kravitz for his second studio album, Mama Said (1991).

Retrieved on August 19, 2007. Kravitz's biggest single yet, "It Ain't Over 'til It's Over", went to number 2 on the Billboard Hot 100.

\begin{tabular}{|c|c|c|c|}
\hline Act & Order & Song & Rock Artist \\
\hline In Stereo & 4 & Demons & $\begin{array}{l}\text { Imagine Drag- } \\
\text { ons }\end{array}$ \\
\hline Cyrus & 5 & It Ain't Over & Lenny Kravitz \\
\hline $\begin{array}{l}\text { Villanueva } \\
\text { Michaela } \\
\text { Baranov }\end{array}$ & 6 & $\begin{array}{l}\frac{\text { til It's Over }}{\text { Wild Horses }} \\
\text { Wild }\end{array}$ & $\begin{array}{l}\text { The Rolling } \\
\text { Stones }\end{array}$ \\
\hline
\end{tabular}

Ronnie Bremer raced the first five races of the season with Brooks Associates Racing, before moving to Polestar Motor Racing.

\begin{tabular}{lll}
\hline Place & Name & Team \\
\hline 5 & Ronnie Bremer & Brooks Associates Racing \\
9 & Bryan Sellers & Lynx Racing \\
9 & Jonathan & Transnet Racing \\
& Bomarito & \\
\hline
\end{tabular}

Donovan also appeared in the $\underline{1980}$ film Breaker Morant, but in a subsidiary role, rather than as the title character.

\begin{tabular}{lll}
\hline Title & Year & Role \\
\hline Cop Shop (TV series) & $1978-$ & Detective Sgt. Vic \\
& 1980 & \\
Breaker Morant & $\underline{1980}$ & Captain Simon Hunt \\
Smash Palace & 1981 & Traffic Officer \\
\hline
\end{tabular}

Try a Little Kindness is the sixteenth album by American singer/guitarist Glen Campbell, released in $\underline{1970}$.

At the height of his popularity, a 1970 biography by Freda Kramer, The Glen Campbell Story, was published.

\begin{tabular}{llll}
\hline Day & Album & Artist & Notes \\
\hline 1 & On the Boards & Taste & - \\
26 & Chicago & Chicago & aka Chicago II \\
- & $\frac{\text { Trya Little }}{\text { Kindness }}$ & Glen Campbell & - \\
& & & \\
\hline
\end{tabular}

Table 10: Pre-training data examples, hybrid setting. 